\title{
Les services pour les Archives Ouvertes: de la référence à l'expertise
}

\section{Introduction}

Les archives ouvertes ou "digital repositories", entendus comme des dépôts numériques de documents scientifiques, sont devenus en vingt ans des dispositifs significatifs de la communication scientifique dans de nombreux domaines [1]. Imaginés pour en être un support à coût minimal, les logiciels d'archives ouvertes se conçoivent d'abord comme des interfaces rudimentaires de dépôt, de collecte et d'accès aux documents [2]. Les professionnels de l'information scientifique et technique s'engagent désormais à dépasser ce stade infrastructurel et à proposer des services associés de seconde génération, orientés utilisateurs finaux en fonction de besoins identifiés, et s'appuyant sur des technologies avancées telles que le text et data mining $[3,4,5,6]$. La question des services à valeur ajoutée a de fait été l'un des enjeux remarqué du congrès Open Repository 2010 (OR2010) [7]. Si ces réflexions sont également pensées pour les archives ouvertes institutionnelles, les dépôts thématiques, nationaux ou inscrits dans des projets européens et internationaux sont pressentis pour supporter prioritairement le développement de services, en ce qu'ils s'adressent à des communautés importantes en nombre et identifiées, qu'ils bénéficient de soutiens financiers et sont les plus à mêmes de permettre des économies d'échelle [8,9].

L'étude présentée se propose, après une première partie sur les principaux enjeux associés au développement de services pour les Archives Ouvertes, de dresser dans une seconde partie un état de l'art des services actuellement disponibles sur les Archives ouvertes. Les dispositifs sélectionnés, répondant à des critères de fiabilité, de masse critique et de couverture géographique, typologique et disciplinaire, ont été étudiés au travers d'une grille d'analyse fonctionnelle (tab. 1). L'étude s'enrichit également des projets présentés lors du congrès OR2010, tenu en juillet 2010 à Madrid sous l'égide du Fécyt [10]. Partant du constat que les services associés aux Archives Ouvertes se déportent peu à peu des seules références et/ou documents pour mettre l'auteur au cœur des données d'information, la troisième partie de l'article interroge en conclusion l'opportunité d'exploiter ces dispositifs pour renouveler les circuits de mise en visibilité et d'appel à contribution des évaluateurs, rapporteurs ou experts d'un domaine scientifique.

\section{Services à valeur ajoutée pour les Archives Ouvertes : quels enjeux ?}

Ce sont les enjeux proprement internes aux dynamiques scientifiques qui nous intéressent ici. L'enjeu premier de l'implémentation de services avancés réside dans la fidélisation des usagers et l'encouragement au dépôt: il s'agit de mieux remplir les objectifs originels de libre accès aux savoirs scientifiques porté par le mouvement de l'Open Access, dans un contexte d'économie de l'attention qui voit se multiplier les dispositifs concurrents et les diffcultés corrélées d'appropriation par les chercheurs ${ }^{1}$. L'idée selon laquelle les Archives Ouvertes peuvent s'articuler avec les modalités actuelles d'évaluation de la recherche, voire les bousculer, mobilise également la réflexion portée sur les services [11]. Ces dispositifs sont aussi pressentis pour assurer avec succès les fonctions de collaboration, et plus largement de facilitation de la communication spécialisée et de co-production de la science [12]. Enfin, et conséquemment, les Archives Ouvertes peuvent être envisagées comme support de visualisation et d'analyse des tendances de la recherche en train de se faire, autant grâce à l'analyse des contenus que par la mise en visibilité des réseaux d'acteurs [13].

\footnotetext{
1 Voir à ce propos l'étude : RESEARCH INFORMATION NETWORK. If you build it, will they come ? How researchers perceive and use web 2.0. RIN, 2010

$<$ http://www.rin.ac.uk/our-work/communicating-and-disseminating-research/use-and-relevance-web20-researchers $>$
} 
Corrélativement à ces objectifs, il nous semble que les Archives Ouvertes, par ce qu'elles sont par nature des "équipements d'authentification" [14] et qu'elles supportent les savoirs experts contributifs autant qu'interactionnels [15], peuvent concourir à renouveler les modalités de repérage d'un expert. La question mérite d'être abordée au titre que les procédures de sélection des experts dans les processus d'évaluation des articles ou des projets de recherche restent aujourd'hui limitées et controversées $[10,16]$. Les enjeux de relégitimation des scientifiques comme figures expertes, en ce sein mais également dans leur interaction avec le reste de la société, sont également à prendre en considération [16, 17].

Il s'agit à présent de voir comment et dans quelle mesure les services installés sur les Archives Ouvertes répondent déjà à ces objectifs.

\section{Etat de l'art des services installés sur les Archives Ouvertes}

L'étude présentée porte sur une sélection de 7 dispositifs répondant à des critères de fiabilité (longévité, porteurs), de masse critique (nombre de dépôts et de déposants), et de couverture géographique, typologique et disciplinaire (dépôts nationaux ou internationaux, thématiques ou pluridisciplinaire, STM ou SHS) (tab. 1): ont été sélectionnées les Archives Ouvertes PubMed Central et UK, HAL-SHS, E-LIS, RePEc et des moissonneurs Driver et Avano. Le réseau social dédié aux scientifiques Research Gate a été adjoint à l'étude en ce qu'il s'adosse aux Archives Ouvertes ${ }^{2}$. C'est déjà, à l'instar des moissonneurs et de RePEc (base de données bibliographiques + modules d'exploitation des références), un outil-service en soi, reposant sur l'accès à des documents open access stockés dans des digital repositories épars.

C'est une approche fonctionnelle qui a été choisie pour analyser ces objets hétérogènes, comme la plus à même d'établir de façon transversale des familles de fonctions et les fontionnalités servicielles associées (tab.1). Le développement qui suit détaille les fonctionnalités identifiées. D'abord les fonctionnalités structurelles d'alimentation, de validation, d'identification, de consultation et d'accession. Puis les fonctionnalités innovantes de personnalisation, de publicisation, de contextualisation des références, de communication et de collaboration entre usagers. Cette description s'enrichit des projets présentés au congrès OR2010 [9].

\section{Alimenter, valider, identifier}

A l'étape de l'alimentation, plusieurs types d'acteurs peuvent intervenir : soit usagers directs (lecteurs, auteurs-déposants, administrateurs alimentant et/ou validant), soit collaborateurs facilitateurs (partenaires éditoriaux, de recherche). Tous peuvent s'appuyer sur divers services d'aide au versement: tutoriels d'utilisation des workflow et/ou masques de saisie avec possibilité de contacter les administrateurs ${ }^{3}$, outils d'aide à conformité des contenus avec les exigences techniques de l'édition électronique (validateurs XML, SGML, etc.), référentiels plus ou moins exhaustifs sur la politique Open Access des éditeurs (lien direct vers SherpaRomeo sur HAL-SHS par exemple) ou des fonds de financement de la recherche (Grant LookUp de PubMed UK). Notons également la médiation institutionnelle, comme dans le cas de RePEc où le MRPA ${ }^{4}$ assure cette fonction pour les auteurs dont l'institution n'est pas affiliée au projet RePEc.

L'archivage nécessite inscription et identification : la gestion des noms des auteurs et

Accès aux ressources RePEc, CiteSeer entre autres.

Voir par exemple la page « Manuscript Submission System » du NIH pour PubMed Central

$<$ http://www.nihms.nih.gov/help/\#slideshow>

4

Munich Personal RePEc Archive <http://mpra.repec.org/> 
de leur affiliation est à cette étape un élément clé, pour ne pas dire problématique (graphies multiples, homonymies, évolutions patronymiques, affiliations plurielles) [18]. Sur HALSHS, des index automatiques permettent à l'usager auteur d'éviter les graphies multiples ou la saisie répétée. Sur E-LIS, il est conseillé de s'inscrire dans le répertoire international IRALIS $^{5}$, à partir duquel est attribué un identifiant par auteur. Dans le cas de l'archive décentralisée RePEc, il ne s'agit pas de gérer ces problématiques en amont, mais de permettre une harmonisation et validation en aval, par l'auteur: le module AuthorClaim propose l'ensemble des graphies possibles du nom auteur, que ce dernier peut ensuite valider ou invalider; une requête est ensuite lancée dans le/s dépôt/s d'origine de l'auteur, en fonction de l'affiliation qu'il indique et il peut alors valider ou invalider les items relevés comme étant ou non de son fait ; les références validées par les auteurs alimentent en retour la base de données centralisée du projet RePEc. Un identifiant auteur est attribué par ce biais, auquel on accède en requêtant un nom dans le module RePEc Author Short-ID Lookup ${ }^{6}$.

Deux des sessions générales du congrès OR2010 étaient consacrées à ces problématiques. Elles reflètent des stratégies diversifiées selon les projets et les acteurs impliqués. Dans le cas $\mathrm{du}$ projet allemand $\mathrm{DOARC}^{7}$, visant une interface commune d'accès à des références et citations provenant de dépôts institutionnels éclatés, c'est une identification et une validation avales par les auteurs qui ont été choisies : le dispositif s'appuiera sur le module AuthorClaim, développé à l'origine pour RePEc et présenté ci-dessus [9a]. Le projet ORCID ${ }^{8}$, qui réunit des partenaires commerciaux (Thomson Reuter, Elsevier, Nature entre autres) et des partenaires institutionnels de type archives ouvertes (arXiv, INSPIRE, ADS, SSRN), vise à constituer un répertoire centralisé d'auteurs auxquels seront attribués des identifiants uniques sur le même principe que les DOI (Digital Objetc Identifier) et en liaison avec la base de données gérée par Crossref [9b]. Enfin, une troisième stratégie, d'ordre national, était présentée : le répertoire national japonais des chercheurs, le KAKENHI, servira de base pour la constitution d'une base harmonisée et l'attribution d'un identifiant unique après curation [9c]. Ces projets rejoignent les diverses initiatives institutionnelles ou privées, locales, nationales ou internationales déjà entreprises en ce sens ${ }^{9}$.

\section{Parcourir, rechercher}

Les tableaux 2 et 3 relèvent les différentes modalités d'accès aux références et/ou documents selon les plateformes.

tab. 2 Modalités de consultation présentes sur les Archives Ouvertes

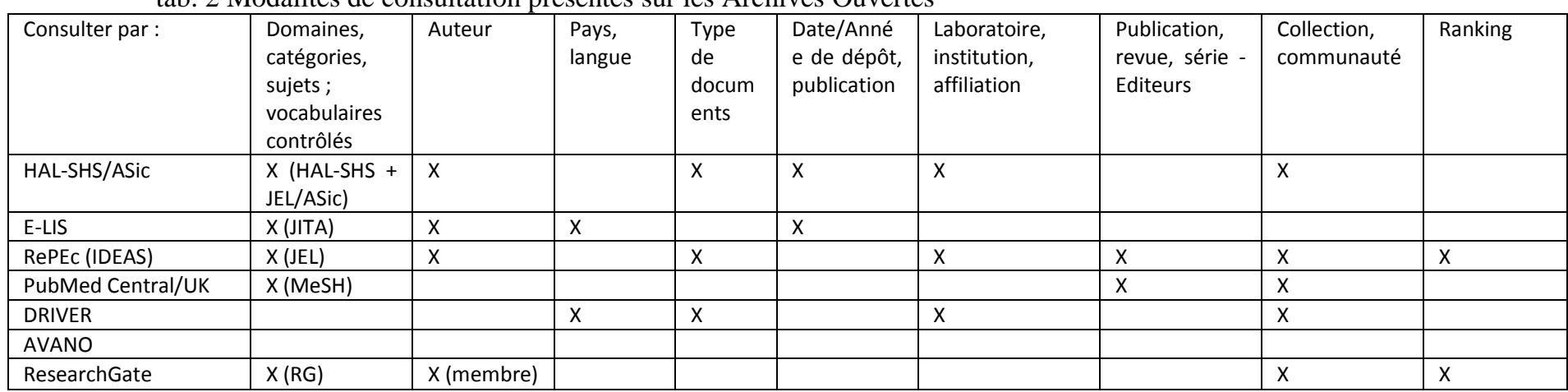

5 International Registry for Authors <http://www.iralis.org/>

6 RePEc Author Short-ID Lookup <http://ideas.repec.org/cgi-bin/shortid.cgi>

7 Distributed Open Access Reference Citations < http://www.isn-oldenburg.de/projects/doarc2/>

8 The Open Researcher and Contributor ID <http://www.orcid.org/>

9 Voir la page Author Identification du wiki International Repositories Infrastructure :

〈http://repinf.pbworks.com/Author-identification > 
tab. 3 : Modalités de recherche présentes sur les Archives Ouvertes

\begin{tabular}{|c|c|c|c|c|c|c|c|c|c|}
\hline Rechercher & $\begin{array}{l}\text { Formulaire } \\
\text { de recherche } \\
\text { simple (mot- } \\
\text { clé) }\end{array}$ & $\begin{array}{l}\text { Formulaire de } \\
\text { recherche } \\
\text { avancée } \\
\text { (équations } \\
\text { booléennes) } \\
\end{array}$ & $\begin{array}{l}\text { Affiner la } \\
\text { recherche } \\
\text { par facette }\end{array}$ & $\begin{array}{l}\text { Recherch } \\
\text { e dans } \\
\text { l'historiqu } \\
\text { e }\end{array}$ & $\begin{array}{l}\text { Equations } \\
\text { complexes, } \\
\text { langage de } \\
\text { recherche } \\
\text { avancé } \\
\end{array}$ & $\begin{array}{l}\text { Aide à la } \\
\text { recherche multi } \\
\text { lingue } \\
\text { (traduction des } \\
\text { requêtes) } \\
\end{array}$ & $\begin{array}{l}\text { Recherche } \\
\text { sémantique } \\
\text { d'un } \\
\text { ensemble } \\
\text { textuel } \\
\end{array}$ & $\begin{array}{ll}\text { Modules de } \\
\text { recherche } \\
\text { spécifique } \\
\text { externes }\end{array}$ & $\begin{array}{l}\text { Recherche } \\
\text { intégrée au } \\
\text { navigateur } \\
\text { (plugin) }\end{array}$ \\
\hline HAL/ASic & $x$ & $x$ & & & & & & & \\
\hline E-LIS & $\mathrm{x}$ & $\mathrm{x}$ & & & & & & & \\
\hline RePEc (IDEAS) & $x$ & $x$ & & & $x$ & $\begin{array}{l}\text { EconomistsOnli } \\
\text { ne }\end{array}$ & & $\mathrm{X}^{* *}$ & $x$ \\
\hline $\begin{array}{l}\text { PubMed } \\
\text { Central/UK }\end{array}$ & $x$ & & $x$ & $x$ & $x$ & & & $\mathrm{X}^{*}$ & $x$ \\
\hline DRIVER & $x$ & $x$ & $\mathrm{X}^{* * *}$ & $x$ & & $x$ & & & \\
\hline AVANO & $x$ & & & $x$ & $x$ & & & & \\
\hline ResearchGate & $x$ & & & & & & $x$ & & \\
\hline
\end{tabular}

Les plateformes proposent des systèmes plus ou moins complexes - au sens de combinatoires (tab. 2 et 3) - d'aide à l'accès à l'information, qui reposent sur des choix documentaires, techniques et technologiques différents.

Les accès par consultation (tab. 2), valorisés sur HAL-SHS, E-LIS et Driver, dépendent essentiellement de la structuration des bases de données, des champs moissonnés et/ou alimentés, et d'une requête préétablie pour consultation ; ce sont ces champs de consultation qui composent les formulaires de recherche avancée (tab. 3). A contrario, c'est la technologie du moteur de recherche qui est privilégiée sur PubMed et Research Gate. Ce dernier vante ainsi la technologie des algorithmes sémantiques : "Are you working on an abstract? Paste a snippet of an abstract and find similar publications through our semantic algorithms. "

La consultation par domaine ou sujet dépend de classements définis en amont, qui peuvent être propres au dispositif, comme le cas des catégories proposées par HAL-SHS ou Research Gate, ou dépendre de classifications partagées par une communauté disciplinaire, comme le code JEL en économie par exemple. L'accès aux publications en série dépend quant à lui des partenariats contractés avec les éditeurs, tel que cela apparaît dans PubMed Central et RePEc (partenariats annoncés avec Elsevier et Springer entre autres).

\section{Personnaliser : veille, gestion documentaire}

A ces modalités d'accès par recherche ou consultation s'ajoutent des fonctionnalités propres à la veille, qui peuvent être intégrées au dispositif ou faire l'objet de modules complémentaires (tab. 4). On peut ainsi créer des alertes par flux rss ou mail dans HAL-SHS, relatives à des domaines, des auteurs et/ou des mots-clés du titre des e-print. Dans Driver et PubMed, il est possible de conserver ses requêtes personnalisées et de les transformer en alertes. Dans le cadre du projet RePEc, le module $\mathrm{NEP}^{10}$ offre de s'abonner à des flux thématiques prédéterminés, alimentés des nouveaux working papers par des experts. Dans le cas de Research Gate, la souscription à des notifications par mail va concerner essentiellement l'aspect social, vie du/de mon réseau (nouveaux membres dans un groupe, nouveaux posts dans un groupe ou d'un membre en particulier, etc) ; mais certaines notifications concernent les publications : nouvelles publications dans une catégorie, un groupe, un profil - et, le plus intéressant peut-être : la possibilité d'être informé lorsqu'une référence demandée devient 
disponible en texte intégral.

Des fonctionnalités de gestion documentaire, plus ou moins avancées selon les dispositifs, complètent encore ces offres de service. Hormis l'export de références bibliographiques et le téléchargement de documents, dont la valeur ajoutée augmente avec la diversité des formats et environnements, les fonctionnalités avancées requièrent la création d'un espace personnel (tab. 4). Non envisagé sur Avano, l'offre de service associée est de fait réduite au minimum. Les fonctions de gestion documentaire sont respectivement nulle et faible sur RePEc d'une part, où la création d'un espace personnel (AuthorPage) est destinée à l'authentification du chercheur et de ses publications, et sur E-LIS d'autre part où l'espace "Your Page » sert uniquement à la gestion des e-prints déposés. De même sur Hal-SHS, où il est possible de configurer des alertes (veille), mais pas de gérer ses propres résultats de requêtes. Des options de conservation et d'organisation en collections sont par contre présentes dans les modules My NCBI (PubMed) et My Driver (Driver). La phase II du projet Driver prévoit plus largement de déployer l'outil ScholarLink [9d], qui est un gestionnaire de référence bibliographique orienté à la fois desktop et web, pensé pour faciliter la constitution de collections partagées. Plus proche encore du travail personnel du chercheur, le plugin MePrint présenté à OR2010 proposera bientôt des fonctions d'analyse des données bibliographiques (occurrence des mots clés) [9e]. Enfin, s'il n'est pas proposé d'organiser ses références en collections sur ResearchGate, il est par contre possible d'adjoindre d'autres références bibliographiques à celles collectées via le dispositif et donc de centraliser ses références. Le service emprunte ici aux agrégateurs participatifs en ligne de références bibliographiques type CiteULike, sans en offrir les fonctionnalités de tagging, propres à la qualification et à l'organisation induites des références.

Tab. 4 Fonctionnalités de gestion des données biographiques et bibliographiques sur les Archives Ouvertes

\begin{tabular}{|c|c|c|c|c|c|c|c|c|c|c|}
\hline \multirow{3}{*}{$\begin{array}{l}\text { Fonction } \\
\text { Fonctionnalités }\end{array}$} & \multirow{4}{*}{$\begin{array}{l}\text { Gestion } \\
\text { personnalisée } \\
\text { Créer un } \\
\text { espace } \\
\text { personnel }\end{array}$} & \multicolumn{3}{|c|}{ Gestion du profil } & \multicolumn{6}{|c|}{ Gestion documentaire } \\
\hline & & \multirow[t]{3}{*}{$\begin{array}{l}\text { Publier des } \\
\text { éléments } \\
\text { biographiques }\end{array}$} & \multicolumn{2}{|c|}{$\begin{array}{l}\text { Publier des éléments } \\
\text { bibliographiques }\end{array}$} & \multicolumn{4}{|c|}{ Gérer ses références et ses documents } & \multicolumn{2}{|c|}{$\begin{array}{l}\text { Gérer ses résultats de } \\
\text { recherche (références } \\
\text { documents) }\end{array}$} \\
\hline & & & \multirow{2}{*}{$\begin{array}{l}\text { Ses } \\
\text { publications }\end{array}$} & \multirow{2}{*}{$\begin{array}{l}\text { Autres } \\
\text { références }\end{array}$} & \multirow{2}{*}{$\begin{array}{l}\text { Déposer, } \\
\text { gérer } \\
\text { ses } \\
\text { dépôts }\end{array}$} & \multirow{2}{*}{ 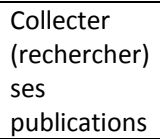 } & \multirow{2}{*}{$\begin{array}{l}\text { Stocker / } \\
\text { uploader } \\
\text { d'autres } \\
\text { références }\end{array}$} & \multirow{2}{*}{$\begin{array}{l}\text { Organiser } \\
\text { en } \\
\text { collections }\end{array}$} & \multirow[t]{2}{*}{ Conserver } & \multirow{2}{*}{$\begin{array}{l}\text { Exporter / } \\
\text { télécharger } \\
\text { (formats) }\end{array}$} \\
\hline $\begin{array}{l}\text { Dispositifs } \\
\text { techniques }\end{array}$ & & & & & & & & & & \\
\hline AVANO & & & & & & & & & & $x$ \\
\hline HAL-SHS/ASic & Mon espace & $\begin{array}{l}\text { Nom-prénom, } \\
\text { affiliation }\end{array}$ & $\begin{array}{l}\text { Page perso } \\
\text { (permalink) }\end{array}$ & & $x$ & $x$ & & & & $x$ \\
\hline E-LIS & Your pages & $\begin{array}{l}\text { Nom-prénom, } \\
\text { affiliation }\end{array}$ & & & $x$ & & & & & $x$ \\
\hline RePEc & Author Page & $\begin{array}{l}\text { Nom-prénom, } \\
\text { affiliations }\end{array}$ & $\begin{array}{l}\text { via } \\
\text { AuthorClaim } \\
\text { (permalink) }\end{array}$ & & & $\begin{array}{l}\text { X } \\
\text { AuthorClaim }\end{array}$ & & & & $\mathrm{x}$ \\
\hline $\begin{array}{l}\text { PubMed } \\
\text { Central/UK }\end{array}$ & My NCBI & username & $\begin{array}{l}\text { My } \\
\text { bibliography } \\
\text { (permalink) }\end{array}$ & $\begin{array}{l}\text { My } \\
\text { bibliography }\end{array}$ & & $x$ & & $\mathrm{x}$ & $x$ & $\mathrm{x}$ \\
\hline DRIVER & My Driver & username & $\begin{array}{l}\text { via } \\
\text { collection } \\
\text { (permalink) }\end{array}$ & $\begin{array}{l}\text { via collection } \\
\text { (permalink) }\end{array}$ & & $x$ & & $\mathrm{x}$ & $\begin{array}{l}\mathrm{X} \\
\text { Dropbox }\end{array}$ & $\mathrm{x}$ \\
\hline ResearchGate & $\begin{array}{l}\text { Home }>M y \\
\text { Profil }\end{array}$ & $\begin{array}{l}\text { Exhaustif, } \\
\text { avec champs } \\
\text { libres (texte) }\end{array}$ & $\begin{array}{l}\text { My } \\
\text { Publications }\end{array}$ & My Library & & $x$ & $\mathrm{x}$ & & $x$ & $\mathrm{x}$ \\
\hline
\end{tabular}




\section{Publiciser}

Ces options documentaires peuvent enrichir, le cas échéant, les pages publiques des membres inscrits aux dispositifs (tab.4). La publicisation, ou mise en visibilité publique volontaire, peut concerner des données biographiques et/ou bibliographiques. Elle peut être interne au dispositif ou externalisée et/ou intégrée à un autre dispositif médiateur grâce à l'attribution d'un permalink (url pérenne). Dans le cas de RePEc, les données biographiques, minimales et de nature institutionnelle, sont complétées par la liste des publications du chercheur, collectée via le module AuthorClaim déjà présenté ; une adresse url pérenne est alors associée à chaque page auteur. Sur PubMed et Driver, un permalink est attribué pour chacune des collections constituées, mais il ne s'agit pas ici de pages publiques internes au dispositif, personnelles et personnalisables. Dans ResearchGate, données biographiques libres, bibliographie auteur (My Publication) et bibliographie générale (My Library) sont au contraire internes et constitutives du dispositif ; seuls les membres inscrits peuvent y accéder directement ou après en avoir fait la demande, selon le paramétrage des droits d'accès au profil. Il est également possible, sur HAL, de constituer une page publique via listing des références déposées et permalink.

\section{Accéder à des documents et/ou des références, des ressources bibliographiques liées et des données primaires liées}

Outre l'accès à des références et/ou des documents, certaines plateformes proposent des accès à des données bibliographiques liées à la référence consultée. Il est ainsi possible sur HALSHS et Driver, contrairement à E-LIS ou Avano, d'accéder à l'ensemble des publications d'un auteur ou de son institution d'affiliation contenues dans le dépôt. Le service EconomistsOnline, adossé aux données de RePEc et enrichi des références d'institutions académiques, donne accès, à partir d'une référence, à toutes les publications d'un auteur, les articles de la revue source, ainsi qu'aux articles indexés avec un même code de la classification JEL. EconPaper (RePEc) indique en outre les références citées dans l'article sélectionné ainsi que les articles le citant (fig. 2). L'ensemble de ces fonctionnalités est proposé dans PubMed Central. Au «link to » s'ajoute ici le «link out», les références renvoyant également à l'ensemble plus vaste PubMed.gov et étant mises en liaison avec les données du portail «Entrez Database ${ }^{11}$ : datasets (bases de données primaires) multiples (GenBank, Nucleotides, etc.), bases de données bibliographiques, sites de référence comme Medline, sites d'autorités (thesaurus, taxonomies). De la même manière, le projet NEEO se propose d'adjoindre, à terme, des accès à des données liées pour les références indexées dans EconomistsOnline ${ }^{12}$.

\section{Accéder à des profils et/ou des «experts»}

Si les références bibliographiques et les documents sont au cœur des enjeux documentaires traditionnels, les offres de services et fonctionnalités visent de plus en plus l'individu, auteur d'un article, expert d'un domaine de recherche et/ou membre actif d'un réseau social. Ce sont des approches servicielles exploitées sur trois des dispositifs étudiés : PubMed, RePEc et Research Gate (tab.1).

Le cas de Research Gate est particulier car, en raison de sa nature de réseau social, son fonctionnement repose, pour moitié, sur les données des profils des membres ; le second pilier réside dans les actions des utilisateurs, qui viennent généralement nourrir en retour les profils (le sien, celui des autres) (tab. 4). L'accès à des profils est ici la raison même du dispositif : les membres ou « researchers » sont considérés comme des ressources à part entière, que l'on peut «bookmarker » (collectionner), annoter (tags, commentaires), contacter. Pour faciliter le

Voir le sitemap du NCBI <http://www.ncbi.nlm.nih.gov/Sitemap/> $<$ http://dvn.iq.harvard.edu/dvn/dv/NEEO> 
push personnalisé via la «technologie des algorithmes sémantiques » - visant la mise en relation avec des membres, des groupes et des références bibliographiques -, les usagers sont invités à exprimer leurs centres d'intérêts en alimentant massivement leur profil.

Dans RePEc, des profils auteurs sont accessibles sur IDEAS et EconPaper. A partir des informations fournies par les auteurs eux-mêmes via le module d'enregistrement Author Service, sont présentés la liste de ses publications, ses affiliations et contacts possibles. Le profil est complété par les codes JEL d'indexation des publications, et les données statistiques de téléchargement et citation obtenus via CitEc et LogEc (fig.2). Le profil est donc constitué à partir d'informations institutionnelles, des données prélevées sur les références bibliographiques et des usages quantifiés associés. Même si le module d'identification Author Service contient un champ de texte libre, les informations formelles dominent largement ; elles offrent une vue sur la présence de l'auteur dans le dispositif mais l'accès aux références bibliographiques reste l'enjeu premier.

Deux modules de l'ensemble PubMed, Authoratory et Scholar Universe, sont quant à eux explicitement présentés comme des banques de données d'experts :

«The information provided on Authoratory.com web site is intended to help visitors to find experts in the specific areas of life-sciences (...). Authoratory.com mission is to identify the experts and help visitors to find and evaluate them.» http://www.authoratory.com/about/about.htm

"Scholar Universe delivers reliable expert search results when searching for qualified experts based on their expertise, location, background, and more.» http://www.scholaruniverse.com/productinfo.jsp

Reposant sur la technique du datamining, des profils automatiques sont constitués à partir des informations disponibles dans une ou plusieurs bases données, agrégeant liste des publications, mentions d'affiliation, contacts possibles (téléphone, mail), co-auteurs, statistiques de téléchargements et de citations, domaines d'expertise via analyse de texte intégral et des termes d'indexation. Les profils Scholar Universe sont signalés, quand ils existent, dans les notices de PubMed par un «link to ». Cependant, la base fonctionne également parallèlement de manière autonome. Il ne s'agit plus, ici, de trouver des références bibliographiques mais des auteurs-experts en tant que tels. Si le service Authoratory est entièrement libre de droit pour les organisations à but non lucratif, l'accès aux données complètes de ScholarUniverse, généré par la société COS-Refworks, est payant. D'autres sociétés privées se positionnent également sur ce qui apparaît comme un "marché de l'expertise ». C'est le cas par exemple de Expernova ${ }^{13}$, qui moissonne les entrepôts OAI$\mathrm{PMH}$ afin de constituer et vendre aux entreprises des banques de données d'experts. 
fig. 1 Accéder à des données bibliographiques, biographiques et statistiques sur EconPaper (RePEc)

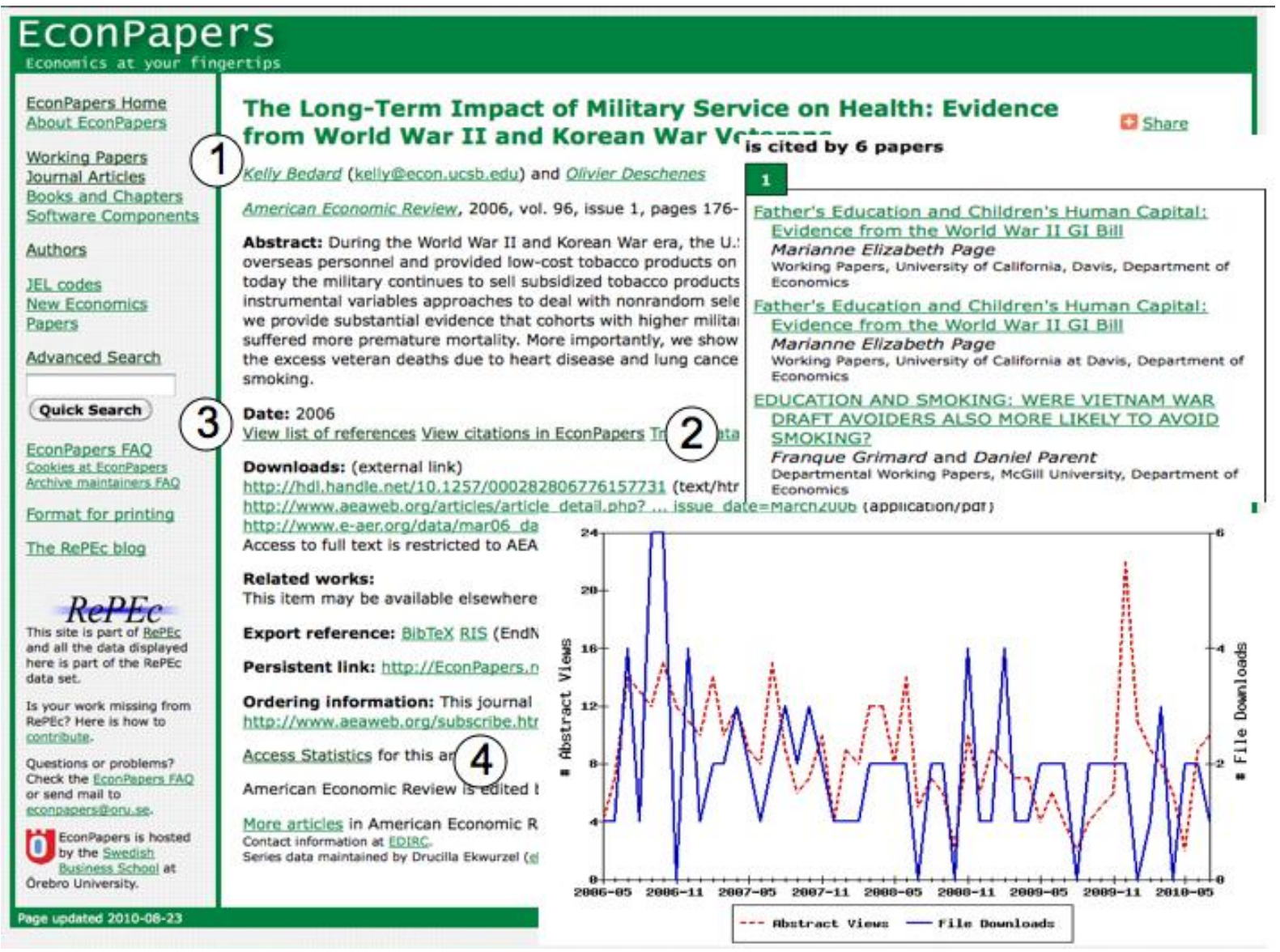

1 Cliquer sur le nom de l'auteur pour accéder à ses contacts, mentions d'affiliation et la liste de ses articles et working papers

2 Voir les références de la base RePEc qui citent cet article (module CitEc)

3 Cliquer pour voir les références citées dans l'article et présentes dans RePEc

4 Voir les statistiques de consultation de cet article (nombre de fois où le résumé a été vu, nombre de téléchargements) (module $\operatorname{LogEc}$ )

\section{Contextualisation vs évaluation}

Les références sont ainsi de plus en plus présentées environnées d'éléments qualitatifs et quantitatifs, en offrant une vue contextualisée : profil plus ou moins exhaustif de l'auteur et des co-auteurs (notamment les affiliations), références citées ou citant le document et statistiques de consultation ont été mentionnés dans les cas de RePEc (EconPaper, fig. 3), PubMed et Research Gate. S'ajoutent dans ce dernier des options de visualisation du réseau de l'utilisateur membre, via un network graph ou carte où sont matérialisées les liaisons entre un membre et ses publications et/ou bibliographies, ses contacts et ses groupes. Des indications de ranking (classement) sont aussi proposées, basées sur les votes des utilisateurs (rating).

La base IDEAS (RePEc) propose également un accès dédié par ranking des auteurs et institutions et des documents les plus cités ou téléchargés ${ }^{14}$. Il procède d'une combinaison entre les données obtenues par les modules LogEc (statistiques de consultation et de téléchargement) et $\mathrm{CitEc}$ (taux de citation). Dans ce cas, on accède aux ressources 
directement à partir du classement : le facteur d'impact l'emporte sur la requête. C'est une logique distincte, qui s'est établie pour concurrencer, ou tout au moins équilibrer, les pratiques controversées d'évaluation s'appuyant principalement sur les données bibliométriques de corpus limités de revues [10].

\section{Communiquer, collaborer}

Une dernière famille de fonctionnalités concerne les fonctions de communication et de collaboration, entre auteurs-lecteurs d'une part et entre membres du dispositif d'autre part. Il est ainsi possible d'entrer directement en contact avec un auteur dans RePEc (IDEAS et EconPaper) et sur PubMed où les adresses mails sont notifiées respectivement sur les pages profils ou sur les notices des références consultées. Dans Driver et ResearchGate, les fonctions de communication et de collaboration sont internes au dispositif, elles permettent aux membres d'entrer en contact, de constituer des groupes et de partager un espace de travail (stockage de fichiers, collections partagées, calendrier partagé).

\section{Conclusion et perspectives}

Cette étude comparée a permis de repérer certaines disparités entre les plateformes étudiées, en termes d'offre de services à valeur ajoutée. Elles sont imputables diversement à la nature ou objectifs primaires des dispositifs (archives ouvertes stricto sensus, moissonneurs, réseaux sociaux), aux techniques documentaires et technologies déployées (qualité des métadonnées, référentiels d'indexation, data ou text mining), à la volonté et capacité portées de développement (équipe dédiée d'E-Print, développements de Thomas Krichel et Christian Zimmermann pour RePEc, soutien du JISC à PubMed UK, etc.), ou encore aux partenariats engagés (avec les éditeurs privés par exemple).

Elle montre plus spécifiquement qu'au-delà de la double fonctionnalité structurelle de recherche et d'accès à des références bibliographiques, les dispositifs tendent vers l'association des logiques d'intégration interne et externe [19] avec des logiques web2.0. Portées à l'internationale pour un champ scientifique donné, ces logiques combinées peuvent, de façon parallèle et complémentaire aux procédures et circuits de l'édition traditionnelle, concourir à l'amélioration de l'expertise scientifique [11]. Il sera intéressant d'observer sous cet angle les modalités d'usage des profils auteurs et des bases de données externalisées d'experts, telles qu'elles sont respectivement proposées sur RePEc et PubMed. On peut également imaginer des approches servicielles tierces, qui donneraient davantage la main aux chercheurs pour connecter l'ensemble de leurs activités de recherche et valoriser leur identité numérique [20]. Mais quelques soient les approches choisies, il convient de s'intéresser aux Archives Ouvertes comme support de mise en visibilité des scientifiques et de leur/s domaine/s d'expertise, à même d'élargir les réseaux établis des habitudes de travail, d'enrichir les comités de lecture et d'aider à la mise en relation d'experts à l'international. Cette perspective pose comme condition de réussite l'exhaustivité des dépôts, renvoyant à la mission première des Archives Ouvertes de libre diffusion des savoirs scientifiques.

\section{Bibliographie}

[1] HELENE BOSC. (2005), Archives ouvertes : quinze ans d'histoire. In : Les Archives Ouvertes : enjeux et pratiques. Guide à l'usage des professionnels de l'information, C. Aubry, J. Janik (eds.), Paris : ADBS. Pp 27-54

[2] THIERRY CHANIER. Archives ouvertes et publication scientifique : comment mettre en place l'accès libre aux résultats de la recherche ? Paris : L'Harmattan, 2004.

[3] JU JINGJIE. Leveraging levels of information services and developping knowledge services. The trend of information services in libraries. Library Management. 2006, vol. 27, n6/7, pp. 354-361 
[4] ROBERT CHAVEZ, GREGORY CRANE, ANNE SAUER, ALISON BABEU, ADRIAN PACKEL, GABRIEL WEAVER. Services make the repository. Journal of Digital Information. 2007, Vol 8, No 2, pp. < https://journals.tdl.org/jodi/article/viewArticle/195/179>

[5] ASSOCIATION OF RESEARCH LIBRARIES. The research library's rôle in digital repository services. ARL, Washington, 2009, 52 p. < http://www.arl.org/bm doc/repository-services-report.pdf>

[6] LES CARR, TIM BRODY. Citation Services (Overview). International Workshop and Co-operation on Citation Services and Repositories. Utrecht, 2010. < http://eprints.ecs.soton.ac.uk/21260/>

[7] PHILIP HUNTER, ROBIN TAYLOR. Report on Open Repository 2010. Ariadne, 2010, $n^{\circ} 64$ $<$ http://www.ariadne.ac.uk/issue64/or-10-rpt/>

[8] DAVE PUPPLET. Subjetc repositories. Ariadne, 2010, n62. <http://www.ariadne.ac.uk/issue62/blsubject-repos-rpt/>

[9] CHRIS ARMBUSTER, LAURENT ROMARY. Comparing repository types. Challenges and barriers for subjectbased repositories, research repositories, national repository systems and institutional repositories in serving scholarly communication. 2009 < http://papers.ssrn.com/sol3/papers.cfm?abstract id=1506905>

[10] FUNDACION ESPANOLA PARA LA CIENCIA Y LA TECNOLOGIA (FECYT), MINISTERO DE CIENCIA Y INNOVACION. OR2010. The 5th International Conference on Open Repositories. Madrid. 6-9 juillet 2010. < http://or2010.fecyt.es>

[10a] MICHAEL MAUNE, EBERHARD R. HILF. Open Access Reference Citations Service with author-identification. Open Repository 2010, general session 1B: Citation \& Bibliography

[10b] SIMEON WARNER. Author identifiers. Open Repository 2010, general session 4A: Data integration and desambiguation

[10c] KEI KURAKAWA, HIDEAKI TAKEDA, AKIKO AIZAWA, MASAO TAKAKU. Researcher Name Resolver: A framework for researcher identification in Japan. Open Repository 2010, general session 4A: Data integration and desambiguation

[10d] GABRIELLA KAZAI, PAOLO MANGHI, ANTONIS LEMPESIS. ScholarLynk: A Reading List Metaphor for Collaborative Research. Poster session:28.

[10e] PATRICK MCSWEENEY. The Semantics of Reading Lists. Eprints Users Group:session 3A

[11] MURIEL LEFEBVRE. L'évaluation des savoirs scientifiques: modalités et enjeux in SCHOPFEL (dir). La publication scientifique : analyses et perspectives, Hermès, 2008, pp. 299-316.

[12] EVELYNE BROUDOUX, GHISLAINE CHARTRON. La communication scientifique face au Web2.0 Premiers constats et analyse. H2PTM'09 - Rétrospective et perspective - 1989-2009. H2PTM, France (2009)

[13] Katy BÖRNER, WEIXIA HUANG, MICAH LINNEMEIER, RUSSEL J. DUHON, PATRICK PHILIPPS, NIANLI MA, ANGELA M. ZOSS, HANNING GUO, MARK A. PRICE. Rete-netzwerk-red: analyzing and visualizing scholarly networks using the Network Workbench Tool. Scientometrics, Budapest, 2010. DOI 10.1007/s11192-009-01490

[14] YVES TREPOS. La sociologie de l'expertise. PUF, Paris, 1996.

[15] HARRY COLLINS, ROBERT EVANS. Rethinking Expertise. Chicago and London, The University of Chicago Press, 2007.

[16] CATHERINE VILKAS. Des pairs aux experts : l'émergence d'un " nouveau management » de la recherche scientifique ? Cahiers internationaux de sociologie. PUF, 2009/1 - 126, pp. 61-79.

[17] ISABELLE BERREBI-HOFFMANN, MICHEL LALLEMENT. A quoi servent les experts ? Cahiers internationaux de sociologie. PUF, 2009, vol. 1, n²126, pp. 5-12. 
[18] DOROTHEA SALO. Name authority control in institutional repositories. Cataloging and Classification Quaterly. 2009, v. 47, n³4, pp. 249-261. ISSN 0163 - 9374.

[19] GHISLAINE CHARTRON. Les chercheurs et la documentation numérique : nouveaux services et usages . Paris: éd. du Cercle de la librairie, 2002.

[20] GABRIEL GALLEZOT, OLIVIER LE DEUFF. Chercheurs 2.0 ? Les Cahiers du Numérique, 2010 